\title{
The Effect of Electrocautery around the Patellar Rim in Patellar Non-Resurfacing Total Knee Arthroplasty
}

\author{
Soo Jae Yim, PhD, Mun Suk Jang, MD, Wook Joong Kim, MD, Sang Hyok Lee, MD and Hee Kyung Kang, MD \\ Department of Orthopedic, Soonchunhyang University Bucheon Hospital, Soonchunhyang University College of Medicine, Buchon, Korea
}

Purpose: The purpose of this study was to evaluate the clinical effect of electrocautery on the reduction of pain in patellar non-resurfacing bilateral total knee arthroplasty.

Materials and Methods: A total of 50 patients were enrolled into this study; all patients had undergone bilateral patellar non-resurfacing total knee arthoplasty at our hospital, between January 2007 to December 2008. The minimum follow-up period was 1 year. The electrocautery of the patellar rim was performed randomly on one side only. The clinical results were evaluated between the electrocautery group and the non-electrocautery group based on measures of anterior knee pain, range of motion, American Knee Society clinical rating score, Feller knee score, Western Ontario and McMaster Universities score, and radiographic analysis.

Results: There were statistically significant differences between preoperative and postoperative status for all parameters. There were no statistically significant differences noted between the electrocautery group and the non electrocautery group for all parameters.

Conclusions: Electrocautery of patellar rim is thought to be less effective in reducing anterior knee pain.

Key words: Patellar rim, Anterior knee pain, Electrocautery, Total knee arthroplasty.

\section{Introduction}

Patient satisfaction has been increasingly considered as an important factor in evaluating the success of a total knee arthoplasty (TKA) for degenerative osteoarthritis ${ }^{1}$. Accordingly, anterior knee pain relief has become one of the primary goals of $\mathrm{TKA}^{2,3}$. Anterior knee pain that is attributed to the patellofemoral joint occurs in $10-15 \%$ of the patients after TKA ${ }^{4,5}$. A reduction in

Received May 27, 2011; Revised (1st) June 26, 2011; (2nd) July 19, 2011; (3rd) October 4, 2011; (4th) November 6, 2011;

(5th) December 21, 2011; (6th) February 23, 2012;

Accepted March 7, 2012.

Correspondence to: Sang Hyok Lee, MD.

Department of Orthopedic, Soonchunhyang University Bucheon

Hospital, Soonchunhyang University School of Medicine, 170 Jomaru-

ro, Wonmi-gu, Bucheon 420-767, Korea.

Tel: +82-32-621-5256, Fax: +82-32-621-5018

Email: sang158@hanmail.net

This is an Open Access article distributed under the terms of the Creative Commons Attribution Non-Commercial License (http://creativecommons.org/licenses/by-nc/3.0/) which permits unrestricted non-commercial use, distribution, and reproduction in any medium, provided the original work is properly cited. anterior knee pain would result in greater patient satisfaction and improved range of motion in TKA patients, especially those in Asia where the cross-legged sitting posture is frequently required for activities of daily living ${ }^{6-8)}$. The impact of patellofemoral joint resurfacing in primary TKA on postoperative knee pain has been controversial ${ }^{4,9,10)}$. van Jonbergen et al. ${ }^{11)}$ employed circumpatellar electrocautery to reduce anterior knee pain in $56 \%$ of the nonresurfaced patellae and in $32 \%$ of the resurfaced patellae in TKA. In addition, Gupta et al. ${ }^{12)}$ investigated the efficacy of electrocautery in anterior knee pain reduction. The purpose of our study was to assess the influence of electrocautery on pain reduction in patients who underwent patellar non-resurfacing bilateral TKA. Our hypothesis was that electrocautery of the patellar rim would affect the postoperative pain in the anterior patella-femoral joint.

\section{Materials and Methods}

Between January 2006 and December 2007, 62 patients underwent patellar non-resurfacing bilateral TKA at our institution. Of these, 12 patients were excluded from this study. The exclusion criteria were the following: $\geq 15^{\circ}$ of varus/valgus, patellar dislocation, or a diagnosis of rheumatoid arthritis. In the 50 enrolled patients, the surgery was performed first on the side 
where the pain was more severe, regardless of the severity of the arthritis, whereas electrocautery was randomly performed on one side regardless of the patellar cartilage condition. The mean age of the patients at the time of surgery was 70.2 years (range, 57 to 84 years). All the study participants were female. The mean follow-up period was 21 months (range, 12 to 48 months). The implanted prosthesis was Nexgen LPS (Zimmer, Warsaw, IN, USA) in all knees $(n=100)$. The preoperative diagnosis was degenerative arthritis in all patients.

The surgery was performed by the same surgeon using a medial parapatellar approach on all patients. After a skin incision, a capsular incision was performed to expose the medial and lateral condyles and the tibial plateau and medial soft tissue release was subsequently carried out. A measured resection technique was used for the proximal tibial and distal femoral bone cuts. The tibial cut was made perpendicular to the mechanical axis using an extramedullary alignment guide, with the posterior tibial slope set at $0^{\circ}$. After thoroughly removing posterior femoral osteophytes, a trial implant was inserted to assess the joint space, varus/valgus stability, patellar tracking, and condylar liftoff. Cement was used for tibial and femoral fixation. Patellar resurfacing was not performed on all knees. Tibial osteophyte removal and chondral shaving were performed. If there was synovial thickening observed in a given patient, a synovectomy was performed as well. Electrocautery was performed on one side in a medial to lateral direction, within $2-3 \mathrm{~mm}$ from the patellar rim. Straight leg raising exercises were initiated from the day of surgery. The drain was removed from the second postoperative day and continuous passive motion was started. From the first postoperative week, weight bearing ambulation was allowed.

Clinical assessment included range of motion, Knee Society knee score and function score, anterior knee pain, quadriceps strength, Feller's patellar score to assess the ability to rise from a chair and climb stairs $\left.{ }^{9}, 13\right)$, and Western Ontario and McMaster Universities (WOMAC) score based on patient's subjective responses ${ }^{14)}$ evaluated before surgery and at last follow-up. For radiographic assessment, Merchant views of the patella were taken before surgery and at the last follow-up to assess changes in the congruence angle and lateral patellofemoral angle.

Statistical analysis was done using SPSS ver 14.0 (SPSS Inc., Chicago, IL, USA). Paired t-tests $(\mathrm{p}<0.05)$ were used to compare the preoperative and postoperative values for the range of motion, Knee Society knee and function score, Feller's patellar score, WOMAC score, congruence angle, and lateral patellofemoral angle. Comparisons between the knees with and without electrocautery of the patellar rim were performed using an independent $\mathrm{t}$-test $(\mathrm{p}<0.05)$.

\section{Results}

\section{Range of Motion}

The mean range of motion was significantly improved from $104.5^{\circ}$ (range, $90^{\circ}$ to $115^{\circ}$ ) preoperatively to $122.6^{\circ}$ (range, $115^{\circ}$ to $130^{\circ}$ ) postoperatively in the electrocauterized knees and from $106.9^{\circ}$ (range, $90^{\circ}$ to $115^{\circ}$ ) to $122.4^{\circ}$ (range, $115^{\circ}$ to $130^{\circ}$ ) in the non-electrocauterized knees. However, there were no notable differences in the range of motion between the 2 groups $(\mathrm{p}=0.133$ ) (Table 1).

\section{Knee Society Clinical Rating Score}

There were significant changes in the preoperative and postoperative Knee Society clinical rating scores regardless of electrocautery use. The mean knee society knee score was changed from 65.62 (range, 53 to 78 ) preoperatively to 86.98 (range, 82 to 95 ) postoperatively in the electrocauterized knees and from 64.54 (range, 52 to 82 ) preoperatively to 86.18 (range, 80 to 93) postoperatively in the non-electrocauterized knees. The mean knee Society function score was changed from 65 (range, 60 to 70 ) preoperatively, to 82.1 (range, 70 to 90 ) postoperatively in the electrocauterized knees, and from 62.7 (range, 50 to 70) preoperatively to 81.4 (range, 80 to 90 ) postoperatively, in the non-electrocauterized knees. However, no remarkable differences were observed between the 2 groups in the Knee Society knee score and function score $(\mathrm{p}=0.853, \mathrm{p}=0.174)$ (Table 1$)$.

\section{Feller's Patellar Score}

The mean Feller's patellar score changed significantly from 16.76 (range, 15 to 19 ) preoperatively to 21.56 (range, 19 to 24) postoperatively, in the electrocauterized knees and from 16.3 (range, 12 to 19 ) preoperatively to 20.56 (range, 18 to 23) postoperatively, in the non-electrocauterized knees. However, there were no significant differences in the Feller's patellar score between the 2 groups $(\mathrm{p}=0.2)$ (Table 2 ).

Table 1. Results of ROM, Knee Score, and Knee Functional Score

\begin{tabular}{lccc}
\hline & $\begin{array}{c}\text { Non electrocautery } \\
\text { group }\end{array}$ & $\begin{array}{c}\text { Electrocautery } \\
\text { group }\end{array}$ & p-value \\
\hline Knee ROM $\left(^{\circ}\right)$ & $106.9 / 122.4$ & $104.5 / 122.6$ & 0.133 \\
Knee score & $64.54 / 86.18$ & $65.62 / 86.98$ & 0.853 \\
Knee function score & $62.7 / 81.4$ & $65 / 82.1$ & 0.174 \\
\hline
\end{tabular}

ROM: range of motion. 
Table 2. Results of Feller Patellar Score, WOMAC Score, Congruence Angle, Lateral Patellofemoral Angle

\begin{tabular}{lccl}
\hline & $\begin{array}{c}\text { Non-electrocautery } \\
\text { group }\end{array}$ & $\begin{array}{c}\text { Electrocautery } \\
\text { group }\end{array}$ & p-value \\
\hline Feller patellar score & $16.3 / 20.56$ & $16.76 / 21.56$ & 0.2 \\
WOMAC score & $58.72 / 26.78$ & $60.46 / 25.4$ & 0.052 \\
Congruence angle & $7.14 / 1.5$ & $7.68 / 1.24$ & 0.296 \\
$\begin{array}{l}\text { Lateral patellofemoral } \\
\text { angle }\end{array}$ & $6.48 / 12.16$ & $6.66 / 11.88$ & 0.057 \\
\hline
\end{tabular}

WOMAC: Western Ontario and McMaster Universities.

A total of 13 patients complained of anterior knee pain after surgery. Five had it on the electrocauterized side, 6 on the nonelectrocauterized side, and 2 on both sides.

\section{WOMAC Score}

The mean WOMAC score was significantly improved from 60.46 (range, 46 to 70 ) preoperatively, to 25.4 (range, 21 to 33) postoperatively, in the electrocauterized knees and from 58.72 (range, 42 to 74 ) preoperatively to 26.78 (range, 20 to 36) postoperatively, in the non-electrocauterized knees. However, there were no significant differences in the WOMAC score between the 2 groups ( $\mathrm{p}=0.052$ ) (Table 2 ).

\section{Radiographic Assessment of the Patella}

There were significant changes in the congruence angle and the lateral patellofemoral angle from the preoperative to postoperative periods regardless of the use of electrocautery. The mean congruence angle and lateral patellofemoral angle were $7.14^{\circ}$ (range, $10^{\circ}$ to $5^{\circ}$ ) and $6.48^{\circ}$ (range, $4^{\circ}$ to $8^{\circ}$ ) preoperatively, and $1.5^{\circ}$ (range, $0^{\circ}$ to $3^{\circ}$ ), $12.16^{\circ}$ (range, $10^{\circ}$ to $15^{\circ}$ ) postoperatively in the electrocauterized knees, whereas the values were $7.68^{\circ}$ (range, $6^{\circ}$ to $11^{\circ}$ ) and $6.66^{\circ}$ (range, $5^{\circ}$ to $8^{\circ}$ ) preoperatively, and $1.24^{\circ}$ (range, $0^{\circ}$ to $3^{\circ}$ ) and $11.88^{\circ}$ (range, $10^{\circ}$ to $15^{\circ}$ ) postoperatively in the non-electrocauterized knees. However, no statistically significant differences were found between the 2 groups in both the congruence angle and lateral patellofemoral angle ( $\mathrm{p}=0.296, \mathrm{p}=0.057$ ) (Table 2).

\section{Complications}

Complications related to patellar fracture, dislocation, subluxation, and extensor mechanism were not observed regardless of the use of electrocautery. No adjuvant or revision surgery due to pain or patellofemoral problems was necessary.

\section{Discussion}

Anterior knee pain in the patellofemoral area after TKA has been addressed in a growing number of studies ${ }^{4,9)}$. Picetti et al. ${ }^{15)}$ reported that anterior knee pain was present in $29 \%$ of the knees after TKA without patellar resurfacing. Unfortunately, many aspects of anterior knee pain after TKA have yet to be fully understood. In addition, patellar resurfacing for anterior knee pain reduction in TKA still remains controversial, because it has been associated with fracture, subluxation and dislocation of the patella, aseptic loosening, and patella necrosis ${ }^{10,16,17}$. In this study, we performed electrocautery of the patellar rim in TKA to relieve pain in the patellofemoral area.

Medial patellar nerve travels within vastus medialis and so does lateral patellar nerve within vastus lateralis ${ }^{18,19}$. In an attempt to interrupt these potential pain pathways, Gupta et al. ${ }^{12)}$ performed patellar rim cauterization. Some authors associated peripatellar soft tissues and infrapatellar fat pad with anterior knee pain ${ }^{20,21)}$. Wojtys et al. ${ }^{22}$ reported that distribution of substance-P nerve fibers in the soft tissues around the knee suggests that anterior knee pain is linked with hyperinnervation of the peripatellar soft tissues.

However, there have been no studies documenting favorable results of patellar rim electrocautery in $\mathrm{TKA}^{12}$. Considering that pain is a subjective symptom, it is difficult to make an accurate assessment on patellofemoral joint pain. Accordingly, the mechanism of anterior knee pain after TKA and the efficacy of patellar rim electrocautery, as a pain reliving measure, have not been clearly elucidated.

Contrary to the study by Gupta et al. ${ }^{12)}$ where the efficacy of electrocautery was evaluated in patients with unilateral TKA, we enrolled patients with patellar non-resurfacing bilateral TKA and performed electrocautery randomly on one side to improve the accuracy of the evaluation.

In this study, electrocautery of the patellar rim in knees with patellar non-resurfacing TKA did not result in differences in the range of motion, Knee Society clinical rating score, Feller's patellar score for assessing symptoms and knee function, WOMAC score for assessing subjective satisfaction, and radiographic results. However, the lateral patellofemoral angle was increased in the knees with electrocautery. This is not congruent with previous reports, which should be investigated in further studies.

The limitations of this study include the small number of patients and the short-term follow-up. In addition, except for patellar rim electrocautery, other factors that may have affected the results were not assessed. The electrocauterization technique 
was not explicitly described, the lateral patellofemoral angle results were not in agreement with those of other parameters, and the patients were not matched for severity of cartilage degeneration.

\section{Conclusions}

The use of electrocautery of patella rim in non-resurfacing bilateral TKA did not result in significant differences in the clinical results, the Feller's patellar score, the WOMAC score, or the radiographic results. Therefore, we believe electrocautery of the patella rim is not effective for relieving anterior knee pain.

\section{References}

1. Ritter MA, Campbell ED. Effect of range of motion on the success of a total knee arthroplasty. J Arthroplasty. 1987;2:957.

2. Kim TK, Kwon SK, Kang YG, Chang CB, Seong SC. Functional disabilities and satisfaction after total knee arthroplasty in female Asian patients. J Arthroplasty. 2010;25:458-64.e1-2.

3. Kohl S, Evangelopoulos DS, Hartel M, Kohlhof H, Roeder C, Eggli S. Anterior knee pain after total knee arthroplasty: does it correlate with patellar blood flow? Knee Surg Sports Traumatol Arthrosc. 2011;19:1453-9.

4. Burnett RS, Boone JL, Rosenzweig SD, Steger-May K, Barrack RL. Patellar resurfacing compared with nonresurfacing in total knee arthroplasty. A concise follow-up of a randomized trial. J Bone Joint Surg Am. 2009;91:2562-7.

5. Holt GE, Dennis DA. The role of patellar resurfacing in total knee arthroplasty. Clin Orthop Relat Res. 2003;(416):76-83.

6. Kim YH, Choi Y, Kim JS. Comparison of a standard and a gender-specific posterior cruciate-substituting high-flexion knee prosthesis: a prospective, randomized, short-term outcome study. J Bone Joint Surg Am. 2010;92:1911-20.

7. Sivarasu S, Mathew L. Biomechanical evaluation of degree of freedom of movements of a novel high-flexion knee for its suitability in eastern lifestyles. J Long Term Eff Med Implants. 2009;19:265-70.

8. Yamazaki J, Ishigami S, Nagashima M, Yoshino S. Hy-Flex II total knee system and range of motion. Arch Orthop Trauma Surg. 2002;122:156-60.

9. Feller JA, Bartlett RJ, Lang DM. Patellar resurfacing versus retention in total knee arthroplasty. J Bone Joint Surg Br. 1996;78:226-8.

10. Fu Y, Wang G, Fu Q. Patellar resurfacing in total knee arthroplasty for osteoarthritis: a meta-analysis. Knee Surg Sports Traumatol Arthrosc. 2011;19:1460-6.

11. van Jonbergen HP, Barnaart AF, Verheyen CC. A dutch survey on circumpatellar electrocautery in total knee arthroplasty. Open Orthop J. 2010;4:201-3.

12. Gupta S, Augustine A, Horey L, Meek RM, Hullin MG, Mohammed A. Electrocautery of the patellar rim in primary total knee replacement: beneficial or unnecessary? J Bone Joint Surg Br. 2010;92:1259-61.

13. Insall JN, Dorr LD, Scott RD, Scott WN. Rationale of the Knee Society clinical rating system. Clin Orthop Relat Res. 1989;(248):13-4.

14. Bellamy N, Buchanan WW, Goldsmith CH, Campbell J, Stitt LW. Validation study of WOMAC: a health status instrument for measuring clinically important patient relevant outcomes to antirheumatic drug therapy in patients with osteoarthritis of the hip or knee. J Rheumatol. 1988;15:1833-40.

15. Picetti GD 3rd, McGann WA, Welch RB. The patellofemoral joint after total knee arthroplasty without patellar resurfacing. J Bone Joint Surg Am. 1990;72:1379-82.

16. Kelly MA. Patellofemoral complications following total knee arthroplasty. Instr Course Lect. 2001;50:403-7.

17. Malo M, Vince KG. The unstable patella after total knee arthroplasty: etiology, prevention, and management. J Am Acad Orthop Surg. 2003;11:364-71.

18. Maralcan G, Kuru I, Issi S, Esmer AF, Tekdemir I, Evcik D. The innervation of patella: anatomical and clinical study. Surg Radiol Anat. 2005;27:331-5.

19. Moller BN, Helmig O. Patellar pain treated by neurotomy. Arch Orthop Trauma Surg. 1984;103:137-9.

20. Lehner B, Koeck FX, Capellino S, Schubert TE, Hofbauer R, Straub RH. Preponderance of sensory versus sympathetic nerve fibers and increased cellularity in the infrapatellar fat pad in anterior knee pain patients after primary arthroplasty. J Orthop Res. 2008;26:342-50.

21. Macule F, Sastre S, Lasurt S, Sala P, Segur JM, Mallofre C. Hoffa's fat pad resection in total knee arthroplasty. Acta Orthop Belg. 2005;71:714-7.

22. Wojtys EM, Beaman DN, Glover RA, Janda D. Innervation of the human knee joint by substance-P fibers. Arthroscopy. 1990;6:254-63. 